\section{CLINICAL ENDOCRINOLOGY FOR} PRACTITIONERS AND STUDENTS

By Laurence Martin, M.D., F.R.C.P., and MarTin HyNes, M.D., M.R.C.P. Second edition. Pp. ix +253 , with 39 illustrations. London: J. and A. Churchill Ltd. 1954. 20 s.

Congratulations are due to the authors of this small and eminently readable book. For its size it covers the vast field of endocrinology very competently, presenting up to date material in a critical manner against the background of accepted teachings. The book has I I chapters, nine dealing with individual endocrine glands and their disorders and two devoted to Frohlich's syndrome and obesity and hormone implantation. Wisely the authors have deliberately excluded diabetes mellitus.

The book is not without fault. It is difficult to understand the inclusion of 'Read's formula' for assessing metabolic rate in the section of hyperthyroidism; the B.M.R. itself is of little diagnostic value in the borderline case and the crude approximation which Read's formula provides must be even less help if not grossly misleading. The rapidly developing domain of the adrenal gland is well surveyed, though perhaps too much space is given to the speculations of the Selye school. On page I 46 normal serum values of sodium and potassium are given as $\mathrm{I} 4 \mathrm{I}$ and five with equivalents per I00 cc. respectively. The confusing conditions of hermaphroditism, pseudohermaphroditism and virilism are lucidly expounded.

This book is appropriate to the occasional endocrinologist giving generous guidance to classical and contemporary work. Its greatest merit is perhaps that, in visiting so many topics, it avoids the synoptic style.

H.K.

\section{ABDOMINAL OPERATIONS}

By Rodney MaIngot, F.R.C.S. Third edition. Pp. xii +580 , with 738 illustrations, $I I$ in colour. London: H. K. Lewis \& Co., Ltd. 1955. £8 ros.

In the seven years which have elapsed since the last edition of this exhaustive work on abdominal surgery, many new ideas have been formulated in this, the general surgeon's most important field. Mr. Rodney Maingot's formidable task of re-editing a new edition has therefore involved a considerable alteration in the book's content, the additions to both text and illustrations outweighing the judicious pruning so that its present size might well have justified a reversion to two volumes. The list of distinguished contributors has been trebled and together with the editor they have achieved the same lucidity and factual precision, unbiased and varied viewpoints which characterized the previous editions; viewpoints which include many quotations from the work of other authors.

Possibly the most outstanding general change in the book is the greater prominence given to what might be described as the non-technical aspects of abdominal surgery. Thus among the many new chapters is one devoted to fluid, electrolyte and nutritional problems and another to the investigation of the jaundiced patient. The same approach characterizes the extension of other sections, such as the management of ulcerative colitis, and an excellent chapter on the complications of gastric surgery which includes authoritative and helpful writing on the post-gastrectomy anaemias and dumping syndrome.

This increase in non-technical considerations does not imply that modifications and advances in operative procedure have been overlooked. New chapters such as those on pelvic exenteration and portal hypertension repeat the clarity of description which characterizes the revised sections, among which those on gastric surgery and the biliary apparatus remain outstanding. Much of this clarity is due to the excellence of the profuse accompanying illustrations.

In a work which aims to represent several viewpoints it would be surprising indeed if there was no cause for critical comment. For instance, the daily intravenous administration of 31 . of normal saline for five days after vagotomy would seem to run the risk of a positive sodium balance. The alternative method of closing a disrupted abdominal wound by buried floss-silk sutures and again the desirability of performing jejunostomy for palliation of inoperable gastric carcinoma might be open to question. In fact the author himself quotes a critical rider to this? last suggestion and so exemplifies the thoughtfuB comments which keep a sense of proportion and balance in a book where variety of opinion might otherwise cause confusion.

It is not possible, nor, indeed, necessary, to mention all the additions, so drastic has been the revision of this work which now surpasses the excellence of the earlier editions. It must surely be the most authoritative and comprehensive book we possess on abdominal operations. G.F.H.

\section{BROMPTON HOSPITAL REPORTS Volume XXII. 1953}

Pp. ix +165 , illustrated. London: Brompton Hospital. I954. I5s.

Originally confined to publications of the members of the staff of the Brompton Hospital, the 'Reports' for this year include for the first time publications by the members of the staff of the London Chest Hospital and the Institute of Diseases of the Chest. Amongst others, a valuable paper is published by Hinson, Moon and Plummer on ' Broncho-pulmonary Aspergillosis,' separating those forms where the presence of the fungus is incidental and of no clinical significance, and the two rarer conditions, firstly where an allergic response to the fungus in the bronchial tree can produce consolidation of the lung and secondly a true septicaemic or pyaemic form.

Another important contribution is by Dr. G. Simon, illustrating the tremendous value of lateral tomograms in demonstrating the exact situation and character of intrapulmonary lesions and also as a help in differentiating mediastinal shadows. 